\title{
EDITORIAL
}

\section{A time to stop, a time to start: high-dose chemotherapy in overweight and obese patients}

Bone Marrow Transplantation (2015) 50, 617-618; doi:10.1038/ bmt.2015.26; published online 2 March 2015

The prevalence of overweight and obesity in US adults exceeds $70 \%{ }^{1}{ }^{1}$ Their sometimes co-occurrence as well as their potential promoting association with malignancy has caused significant concern and controversy among research scientists and clinical oncologists, especially those responsible for treating patients with chemotherapy. Cancer chemotherapy is most commonly administered on a weight basis, calculated either directly per unit weight (pound or $\mathrm{kg}$ ) or indirectly relative to body surface area $\left(\mathrm{BSA} ; \mathrm{m}^{2}\right.$ ), which is determined on the basis of height and weight. These practices have evolved from observations such as that in vitro tumor cell killing is dose dependent, higher doses reduce emergence of resistant tumor cell clones and the use of weight and/or BSA-based dosing provides an approach to translate to humans effective doses determined in animals. ${ }^{2,3}$ Moreover, the success associated with high dose, compared with conventional, chemotherapy in hematolymphocytic malignancies provides strong support for the antitumor and the survival benefits of exposing tumors to high concentrations of chemotherapy. ${ }^{4}$

For overweight or obese cancer patients, the use of actual body weight or BSA for chemotherapy dose calculation will result in increased administration of cytotoxic agents relative to patients of normal weight. Because of the concerns that overweight or obese patients may have intrinsically compromised health status, adverse prognostic factors, altered pharmacokinetics and/or significantly impaired survival, and that increased doses of cytotoxic agents will cause greater toxicity, cancer chemotherapy in obese patients has frequently been administered on the basis of ideal weight rather than actual weight, or in some cases, at reduced doses. ${ }^{5-7}$ Unfortunately, these practices may compromise the curative potential of chemotherapeutic agents. An expert panel convened by the American Society of Clinical Oncology (ASCO) reviewed these concerns and the relevant literature, and concluded that the evidence was not sufficient to indicate the occurrence of short- or long-term toxicity among obese patients receiving full weight-based chemotherapy doses. For conventional chemotherapy, the panel recommended using full weight-based dosing, especially when the goal of treatment is cure. ${ }^{8}$

As noted above, the ASCO panel recommendation for full weight-based dosing was for conventional chemotherapy, whereas no recommendations were made for high-dose chemotherapy accompanied by hematopoietic stem cell transplant (HCT) interventions, where similar concerns and controversies exist regarding high-risk prognostic factors, and adverse effects in overweight and obese patients. As a result of these concerns, some patients who might benefit from high-dose therapy are prevented from receiving therapy altogether or they may receive reduced dosing relative to body mass. Although results for efficacy and tolerability of high-dose chemotherapy with HCT in overweight patients are inconsistent, the prevailing concept is that high-dose chemotherapy and HCT can be safely administered in overweight and obese patients without increased adverse effects or compromised outcomes. ${ }^{9-11}$ In a recent perspective, we recommended dosing on the basis of adjusted body weight (Adj
BW) to partially increase the dose relative to increased body mass and to then conduct studies to further increase dosing until full body weight is used as the basis for calculation, or dose-limiting toxicity is reached. ${ }^{12}$ After a comprehensive literature review, the American Society of Blood and Marrow Transplantation Practice Guidelines Committee subsequently concluded that there was insufficient data to make evidence-based recommendations for how to dose high-dose chemotherapy in obese patients. ${ }^{13}$ They, however, provided a series of dosing recommendations for specific chemotherapeutic agents which for different agents were based on either total body weight (TBW) or Adj BW (ideal body weight (IBW) plus 25 or $40 \%$ of (TBW - IBW)) thus employing increased drug in proportion to weight.

In this issue of Bone Marrow Transplantation, Lau et al. ${ }^{14}$ report that the use of Adj BW to calculate the dose of chemotherapy did not adversely impact the outcome in obese lymphoma patients undergoing autologous stem cell transplant using $\mathrm{BU}, \mathrm{CY}$ and etoposide as the myeloablative conditioning regimen. In fact, obese patients who presumably received the highest amounts of chemotherapeutic agents showed a decrease in 100-day mortality relative to normal or overweight patients, but no significant difference in relapse-free survival or OS. ${ }^{14}$

In this single-center, retrospective study of the safety and efficacy of auto-SCT in 476 adult lymphoma patients, all drugs were dosed on the basis of Adj BW in which $25 \%$ of the difference between TBW and IBW was added to the patient's IBW to determine Adj BW (Adj BW=IBW+0.25 (TBW - IBW)), which was then used as the basis for dose calculations.

Compared with other sometimes contradictory reports, important advantages of this single-center report is its size, reporting on 472 patients, and the presumption that all anthropomorphic measurements, all dosing-based calculations and all other aspects of patient management and evaluation were performed in a consistent fashion.

In considering the results of this study, it is important to acknowledge that the goal of the intervention is tumor ablation, not myeloablation. Tumor ablation is expected to be dose dependent, whereas myeloablation is the life threatening severe toxic side effect, which is expected to be bypassed or circumvented by infusion of sufficient autologous stem cells to reconstitute the host hematopoietic capacity. Thus, the restoration of hematopoietic cell function with autologous stem cells is expected to be dependent on the number of infused stem cells and independent of administered drug dose. The efficacy of this approach in circumventing toxicity associated with adjustable weight dosing is clearly shown by the fact that days to recovery of absolute neutrophil count $>500$ cells $/ \mu \mathrm{L}$ and days to recovery of platelets $>20000$ cells $/ \mu \mathrm{L}$ showed no statistical differences between normal/underweight, overweight and obese patients.

In terms of short-term toxicity to other organs, none of the weight-adjusted doses of chemotherapy caused sinusoidal obstructive syndrome, and there was no significant difference by weight group in mucositis score or severe mucositis. Secondary malignancy, a potential long-term toxicity that might be expected to increase with increasing doses of chemotherapy, showed no significant differences associated with weight in 5-year cumulative incidence, and there was no development of any unique type of malignancy. ${ }^{14}$ 
No specific anatomical or molecular markers of post-transplant residual tumor mass are available to determine specific antitumor effects of adjusted weight antitumor therapy. However, as the relapse rates in the three weight groups, at 1, 3 and 5 years, were not significantly different, these results suggest the probability that the increased drug dose associated with obesity was at least as effective in attaining the same degree of tumor control as the more standard dose given to normal weight patients. These results could be interpreted to suggest that drug based on ideal weight in the obese patients might have been inadequate to attain this same degree of tumor control.

On the basis of the results of several retrospective reviews of high-dose chemotherapy that were unable to provide convincing evidence to support the notion that high-dose therapy with HCT in overweight and obese patients is associated with adverse prognostic factors or worse outcomes, it is time to stop performing retrospective literature reviews and to start performing carefully monitored dose-escalation studies to provide overweight and obese patients with maximal drug dose to enhance possibility for complete tumor eradication and cure. For oncologists who remain concerned about full weight-based dosing, this goal could be approached by randomizing patients to dose escalation on the basis of Adj BW to include IBW plus 25, 50, 75 and $100 \%$ of the difference between IBW and TBW. Such studies should include careful monitoring of pharmacokinetics and clinical outcomes including antitumor effects, short- and long-term toxicities, time to relapse and OS.

The report in this issue by Lau et al. ${ }^{14}$ is a step in the right direction and provides a solid base that the floor for escalation of the $\mathrm{BU}, \mathrm{CY}$ and etoposide regimen accompanied by autologous HCT in overweight and obese lymphoma patients should be dosed using Adj BW in which IBW is increased by $25 \%$ of difference between IBW and TBW. It needs to be emphasized that the results reported in this study are specific for the $B U, C Y$ and etoposide regimen and need to be independently established for other agents.

And although not the focus of this commentary, it is noteworthy that obesity is frequently accompanied by inactivity, which in itself is a risk factor for increased mortality. ${ }^{15,16}$ Accordingly, it is probable that the outcomes of high-dose chemotherapy in overweight and obese patients could be improved by institution of vigorous physical activity and exercise programs, before, during and after high-dose chemotherapy. ${ }^{17-19}$

\section{CONFLICT OF INTEREST}

The author declares no conflict of interest.

\author{
NA Berger ${ }^{1,2}$ \\ ${ }^{1}$ Center for Science, Health and Society, Case Western Reserve \\ University School of Medicine, Cleveland, $\mathrm{OH}$, USA and \\ ${ }^{2}$ Case Comprehensive Cancer Center, Case Western Reserve University \\ School of Medicine, Cleveland, $\mathrm{OH}$, USA \\ E-mail:nab@case.edu
}

\section{REFERENCES}

1 Flegal KM, Carroll MD, Kit BK, Ogden CL. Prevalence of obesity and trends in the distribution of body mass index among US adults, 1999-2010. JAMA 2012; 307: 491-497.

2 Hunter RJ, Navo MA, Thaker PH, Bodurka DC, Wolf JK, Smith JA. Dosing chemotherapy in obese patients: actual versus assigned body surface area (BSA). Cancer Treat Rev 2009; 35: 69-78.

3 Lyman GH, Sparreboom A. Chemotherapy dosing in overweight and obese patients with cancer. Nat Rev Clin Oncol 2013; 10: 451-459.

4 Schmitz N, Pfistner B, Sextro M, Sieber M, Carella AM, Haenel M et al. Aggressive conventional chemotherapy compared with high-dose chemotherapy with autologous haemopoietic stem-cell transplantation for relapsed chemosensitive Hodgkin's disease: a randomised trial. Lancet 2002; 359: 2065-2071.

5 Navarro WH, Agovi MA, Logan BR, Ballen K, Bolwell BJ, Frangoul H et al. Obesity does not preclude safe and effective myeloablative hematopoietic cell transplantation (HCT) for acute myelogenous leukemia (AML) in adults. Biol Blood Marrow Transplant 2010; 16: 1442-1450.

6 Lopes-Serrao MD, Ussery SM, Hall RG 2nd, Shah SR. Evaluation of chemotherapyinduced severe myelosuppression incidence in obese patients with capped dosing. J Oncol Pract 2011; 7: 13-17.

7 Tarella C, Caracciolo D, Gavarotti P, Argentino C, Zallio F, Corradini P et al. Overweight as an adverse prognostic factor for non-Hodgkin's lymphoma patients receiving high-dose chemotherapy and autograft. Bone Marrow Transplant 2000; 26: 1185-1191.

8 Griggs JJ, Mangu PB, Anderson H, Balaban EP, Dignam JJ, Hryniuk WM et al. Appropriate chemotherapy dosing for obese adult patients with cancer: American Society of Clinical Oncology clinical practice guideline. J Clin Oncol 2012; 30: 1553-1561.

9 Deeg HJ, Seidel K, Bruemmer B, Pepe MS, Appelbaum FR. Impact of patient weight on non-relapse mortality after marrow transplantation. Bone Marrow Transplant 1995; 15: 461-468.

10 Jaime-Perez JC, Colunga-Pedraza PR, Gutierrez-Gurrola B, Brito-Ramirez AS, Gutierrez-Aguirre H, Cantu-Rodriguez OG et al. Obesity is associated with higher overall survival in patients undergoing an outpatient reduced-intensity conditioning hematopoietic stem cell transplant. Blood Cells Mol Dis 2013; 51: 61-65.

11 Nikolousis E, Nagra S, Paneesha S, Delgado J, Holder K, Bratby L et al. Allogeneic transplant outcomes are not affected by body mass index (BMI) in patients with haematological malignancies. Ann Hematol 2010; 89: 1141-1145.

12 Weiss BM, Vogl DT, Berger NA, Stadtmauer EA, Lazarus HM. Trimming the fat: obesity and hematopoietic cell transplantation. Bone Marrow Transplant 2013; 48: 1152-1160.

13 Bubalo J, Carpenter PA, Majhail N, Perales MA, Marks DI, Shaughnessy P et al. Conditioning chemotherapy dose adjustment in obese patients: a review and position statement by the American Society for Blood and Marrow Transplantation practice guideline committee. Biol Blood Marrow Transplant 2014; 20: 600-616.

14 Lau J, Webber C, Earl M, Rybicki L, Carlstrom K, Wenzell C et al. Outcomes after autologous stem cell translplantation in lymphoma patients grouped by weight Bone Marrow Transplant (e-pub ahead of print 9 February 2015; doi: 10.1038/ bmt.2014.327).

15 Thompson CL, Owusu C, Nock NL, Li L, Berger NA. Race, age, and obesity dis parities in adult physical activity levels in breast cancer patients and controls. Front Public Health 2014; 2: 150

16 Patel AV, Bernstein L, Deka A, Feigelson HS, Campbell PT, Gapstur SM et al. Leisure time spent sitting in relation to total mortality in a prospective cohort of US adults. Am J Epidemiol 2010; 172: 419-429.

17 Wiskemann J, Dreger P, Schwerdtfeger R, Bondong A, Huber G, Kleindienst N et al Effects of a partly self-administered exercise program before, during, and after allogeneic stem cell transplantation. Blood 2011; 117: 2604-2613.

18 Wolin KY, Ruiz JR, Tuchman H, Lucia A. Exercise in adult and pediatric hematological cancer survivors: an intervention review. Leukemia 2010; 24: 1113-1120.

19 Sanchis-Gomar F, Lucia A, Yvert T, Ruiz-Casado A, Pareja-Galeano H, Santos-Lozano A et al. Physical inactivity and low fitness deserve more attention to alter cancer risk and prognosis. Cancer Prev Res (Phila) 2015; 8: 105-110. 